\title{
Nippostrongylus rauschi n. sp. Nématode parasite de Dermoptères et considérations sur $N$. brasiliensis parasite cosmopolite des Rats domestiques
}

\author{
Par Alain-G. CHABAUD et Marie-Claude DESSET
}

Deux Dermoptères Cynocephalus volans (L.), dont le lieu de capture est inconnu, ont été expédiés des Iles Philippines aux Etats-Unis et sont morts en cours de route. Ils ont été autopsiés par notre excellent collègue Robert L. Rausch, de l'Arctic Health Research Center à Anchorage, et celui-ci a eu l'amabilité de nous confier les Helminthes pour étude.

L'intestin grêle des Dermoptères contenait quelques spécimens de Trichostrongylides (un mâle et une femelle chez l'un, un mâle et deux femelles chez l'autre), dont voici la description.

\section{Deseription}

Corps de couleur rougeâtre, enroulé de façon dextre sur environ six tours de spire. Vésicule céphalique bien marquée, haute de $95 \mu$. Cuticule ornée de quatorze arêtes longitudinales naissant derrière la vésicule céphalique et ne s'estompant que tout à fait à l'arrière du corps. La taille des arêtes décroît de façon assez régulière depuis les axes latéraux jusqu'aux axes médians. Sur coupe transversale, on constate que toutes les épines, sauf les deux ventrales, ont leur pointe dirigée vers la face dorsale gauche. Ce système d'arêtes, bien que relativement peu spécialisé, paraît efficace pour assurer la fixation du Ver dans la muqueuse (cf. Desset, 1964). Une étude détaillée en sera faite ultérieurement par l'un de nous. Bouche triangulaire. Cavité buccale très faible, rendue perceptible par l'existence d'un petit renforcement chitinoïde à parois très minces, haut d'environ $4 \mu$, et constituant en vue apicale un triangle arrondi. Esophage augmentant régulièrement de diamètre d'avant en arrière.

Mâle: corps long de $8,2 \mathrm{~mm}$, large de $110 \mu$. Esophage long de $450 \mu$. Anneau 


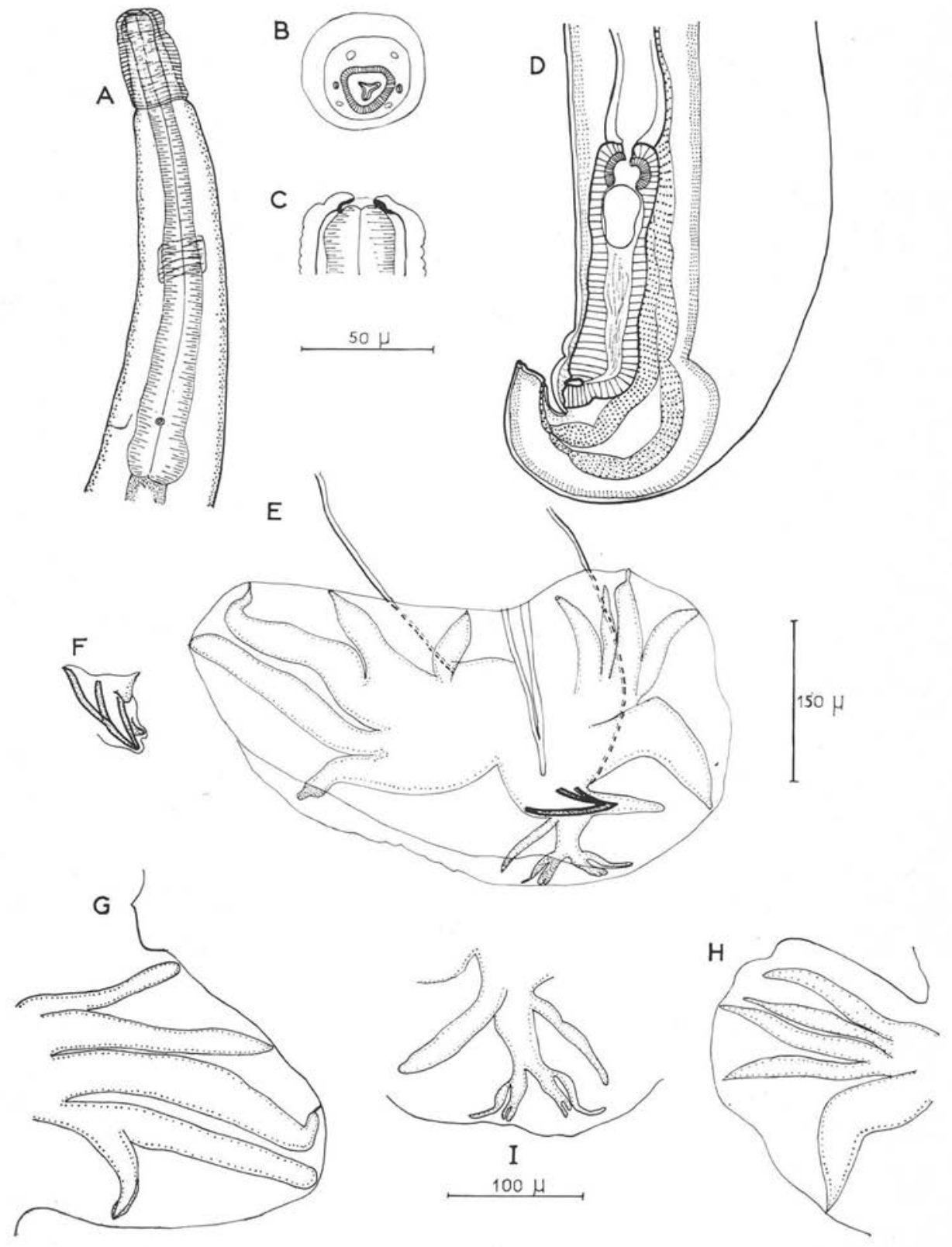

Fig. 1. - Nippostrongylus rauschi. A) Partie antérieure du mâle, vue latérale. B) Tête de la femelle, vue apicale. C) Région antérieure du mâle, vue latérale. D) Extrémité postérieure de la femelle, vue latérale. E) Bourse caudale du mâle, vue ventrale. F) Gubernaculum. G) Lobe droit de la bourse caudale, vue dorsale. $\mathrm{H}$ ) Lobe gauche de la bourse caudale, vue dorsale. I) Lobe dorsal de la bourse caudale, vue dorsale. A, D, E, G, H : $150 \mu ;$ B, C : $50 \mu ;$ F, I : $100 \mu$ 
nerveux, diérides et pore excréteur respectivement à $240 \mu, 390 \mu$ et $405 \mu$ de l'apex. Spicules simples et fins, subégaux, longs de $700 \mu$.

Bourse fortement asymétrique ; le lobe dorsal n'est pas distinct des lobes latéraux, et les deux lobes latéraux fusionnent sur la ligne ventrale; l'ensemble constitue ainsi un cône complet qu'il est impossible de mettre à plat. La figure en vue ventrale donne donc une impression inexacte car certaines côtes, et en particulier les ventrales, sont vues verticalement et paraissent trop trapues. Les figures partielles $\mathrm{G}, \mathrm{H}$ et $\mathrm{I}$ indiquent la longueur réelle des différents éléments. Contrairement à la plupart des Trichostrongyles, c'est le lobe latéral droit qui est hypertrophié (1).

Cependant, la côte postéro-latérale gauche et, à un moindre degré, la côte externodorsale gauche sont nettement plus fortes que les éléments droits correspondants. Le cône génital est renforcé par un gubernaculum de forme complexe (fig. 1, F).

Femelle: corps long de 10,2 mm, large au milieu du corps de $125 \mu$. En arrière, dans la région de l'ovéjecteur et sur une hauteur d'environ $1 \mathrm{~mm}$, la cuticule s'écarte du corps et ménage une vaste dilatation dorsale, atteignant un diamètre d'environ $200 \mu$.

Esophage long de $470 \mu$. Anneau nerveux, diérides et pore excréteur respectivement à $240 \mu, 380 \mu$ et $400 \mu$ de l'apex. Queue très courte $(30 \mu)$ munie d'un petit mucron terminal. Vulve à $60 \mu$ en avant de l'anus. Ovéjecteur figuré en 1, D. Monodelphie. Les œufs contenus dans l'ovéjecteur paraissent ne pas être complètement mûrs ; ils mesurent $60 \mu \times 35 \mu$.

\section{Discussion}

La seule espèce qui soit proche de la forme décrite ci-dessus est Nippostrongylus brasiliensis (Travassos, 1914). L'ornementation cuticulaire, l'anatomie générale, l'enroulement du corps selon une spire dextre, l'asymétrie de la bourse caudale avec hypertrophie du lobe droit et de la côte postéro-latérale gauche sont identiques et témoignent d'affinités étroites entre les deux espèces.

Cependant, les parasites de Dermoptères sont plus grands. La bourse caudale forme un cône complet et continu et le lobe dorsal n'est pas nettement individualisé et de taille réduite comme il l'est chez $N$. brasiliensis (fig. 3). L'extrémité postérieure de la femelle est enflée, mais il n'y a pas invagination et formation d'un prépuce cuticulaire comme on l'observe chez l'espèce type.

Nous pensons donc que le Nématode de Cynocephalus doit être considéré comme une deuxième espèce du genre Nipponstrongylus et nous proposons de le nommer Nippostrongylus rauschi $\mathrm{n}$. sp.

(1) Ceci est lié à l'enroulement dextre du Ver. Au cours de la copulation, le mâle s'enroule autour du corps de la femelle et le lobe postérieur (gauche si l'enroulement est sénestre, droit si l'enroulement est dextre) a plus de surface à couvrir, car c'est lui qui clôt la spire en arrière. Le phénomène est identique chez les nombreux Spirurida où l'extrémité postérieure effectue quelques tours de spire. C'est habituellement une spire sénestre et, lorsque la bourse caudale est asymétrique, c'est le lobe gauche qui est hypertrophié (voir par exemple Viguiera viduae ou Viguiera dorsti, in Chabaud 1960). De même, on remarquera chez les Spirurida que c'est le spicule gauche qui est presque toujours le plus long. 


\section{Considérations sur $N$. brasiliensis}

$N$. brasiliensis est un parasite des Rongeurs domestiques, Rattus rattus, $R$. norvegicus, plus rarement $M$ us musculus et il est considéré, à juste titre actuellement, comme un parasite très cosmopolite. L'histoire de ce Ver semble cependant différente de celle des autres Helminthes d'animaux inféodés à l'homme.

Lutz en 1894 signale à Sao Paulo un Strongle que Travassos reconnaît ultérieurement (1921) être $N$. brasiliensis. En 1914, Travassos trouve et décrit l'espèce à Rio de Janeiro. Mais, en dehors du Brésil, il faut attendre la fin de la première guerre mondiale pour que Nippostrongylus soit retrouvé dans des régions de plus en plus variées et, semble-t-il, au début, presque toujours à proximité de ports importants : à Brisbane en Australie (Johnston, 1918), à Baltimore aux Etats-Unis (Yokogawa, 1921), à Londres en Europe (Dudgeon, 1922). Dans le Pacifique, où les enquêtes sur les Helminthes de Rats sont relativement peu nombreuses, le Ver est trouvé dès 1923 par Lane aux Iles Samoa et en 1931 aux Iles Philippines par Tubangui.

A partir de 1931, ce Nématode est utilisé couramment pour des expériences de physiologie ou d'immunologie dans de nombreux laboratoires, en même temps qu'il est signalé comme parasite spontané dans des régions très variées: ainsi que l'indique le tableau publié par Haley (1961), les pays atteints sont la Chine en 1930, Cuba en 1936, le Japon en 1938, le Canada en 1948, Panama en 1950, l'Espagne en 1957 (1).

Au cours de ces dernières années, le tableau de Voelckel et coll. (1964) indique la découverte du Ver en Tunisie (1963), en Belgique (1963), en France (1964) et Wertheim (1963), le signale en outre en Israël. En fait, certains auteurs estiment que le parasite est maintenant trop cosmopolite pour qu'il soit nécessaire de signaler sa présence en de nouvelles régions. Nous-mêmes l'avons identifié chez des Rattus rattus récoltés, en novembre 1960, par E. Brygoo, à Tananarive.

$\mathrm{Ce}$ bref historique indique donc nettement que l'extension mondiale de Nippostrongylus brasiliensis est de date tout à fait récente. Localisé quelque part dans l'hémisphère Sud et découvert au Brésil vraisemblablement dès 1894, il semble ne s'être répandu largement qu'au moment de la première guerre mondiale et n'avoir atteint la région méditerranéenne qu'au cours de ces dernières années.

Il paraît donc intéressant de découvrir une deuxième espèce extrêmement proche chez un hôte dont la distribution géographique est, cette fois, étroitement limitée.

On constatera en outre que $N$. rauschi a une morphologie plus primitive que celle de $N$. brasiliensis. La réduction du lobe dorsal est moins marquée et l'extrémité postérieure de la femelle est presque identique à celle de la femelle juvénile de brasiliensis (voir la figure 31, planche XI de Yokogawa, 1922) et non à celle de la femelle adulte.

En conclusion, la découverte de $N$. rauschi (espèce extrêmement proche de $N$. brasiliensis, mais légèrement plus primitive) chez un hôte à localisation géographique

(1) Peut-être existait-il en Egypte depuis longtemps car Boulenger (1926) l'identifie dans les collections de Looss; cette référence ne peut cependant être admise sans réserve car, d'une part, la provenance du Rat n'est pas indiquée et, d'autre part, les spécimens sont en trop mauvais état pour que Boulenger puisse affirmer la détermination. 

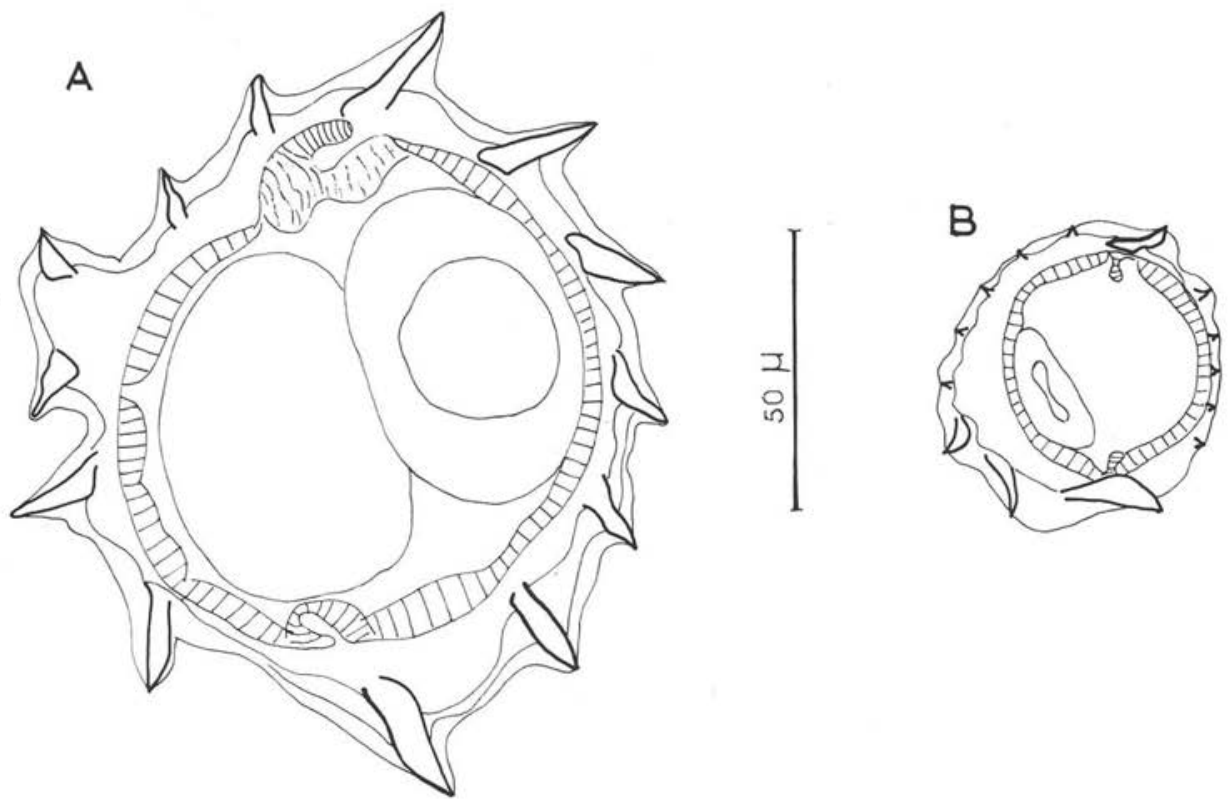

Fig. 2. - Coupes transversales du corps. A) Nippostrongylus rauschi. B) Nippostrongylus brasiliensis

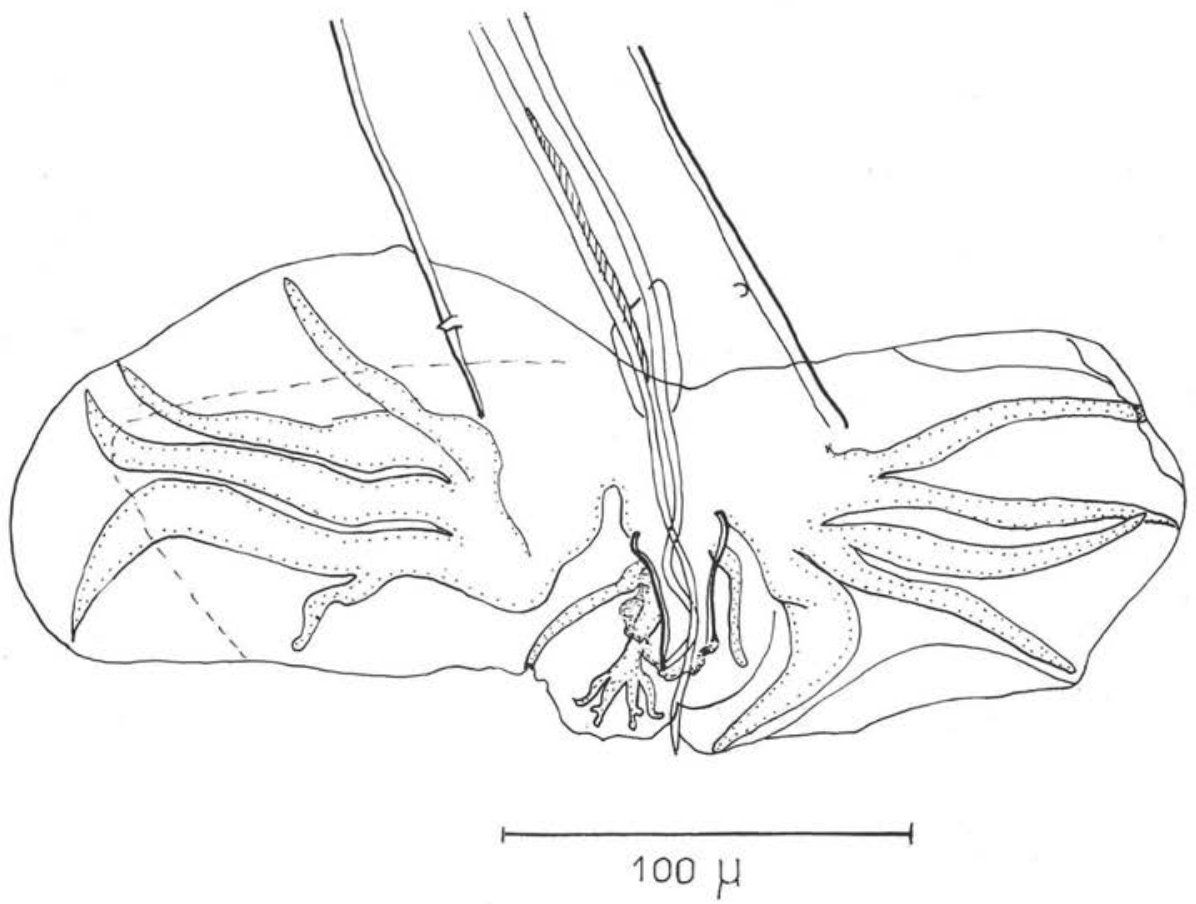

Fig. 3. - Nippostrongylus brasiliensis. Bourse caudale du mâle, vue ventrale 
étroite, jointe à la notion que $N$. brasiliensis n'a acquis son expansion mondiale qu'au cours de ces dernières années, nous incite à croire que le parasite des Rats est un parasite de capture ayant la région des Philippines pour point d'origine.

\section{Résumé}

Nippostrongylus rauschi n. sp., parasite du Dermoptère Cynocephalus volans, est . très proche de $N$. brasiliensis (Travassos, 1914), mais il est plus primitif. Chez le mâle, la côte dorsale est moins réduite; chez la femelle, l'extrémité postérieure n'est pas invaginée et n'a pas de prépuce cuticulaire comme la femelle mûre de $N$. brasiliensis.

La bibliographie semble indiquer que l'extension mondiale de $N$. brasiliensis s'est effectuée très récemment, postérieurement à la première guerre mondiale. Nous pensons que l'espèce des Rongeurs domestiques est un parasite de capture ayant son origine dans la région des Philippines.

\section{Bibliographie}

BoUlenger (C. L.), 1926. - Reports on a collection of parasitic nematodes mainly from Egypt. Part. 4 Trichostrongylidae and Strongylidae. Parasitology, 18, 86-100.

Chabaud (A.-G.), 1960. - Quatre Spirurides parasites d'Oiseaux malgaches. Mémoires de l'Institut Scientifique de Madagascar, série A, 14, 105-124.

Desset (M.-C.), 1964. - Les systèmes d'arêtes cuticulaires chez les Nématodes Héligmosomes. Etude de cinq espèces parasites de rongeurs de La Maboké. Cahiers de La Maboké, 2, 39-78.

Dudgeon (G. C.), 1922. - Occurrence of Heligmosomum brasiliense Trav. in England. Parasitology, 14, 13.

HALEY (A. J.), 1961. - Biology of the rat nematode Nippostrongylus brasiliensis (Travassos, 1914). I. Systematics, hosts and geographic distribution. J. Parasitol., 47, 727-732.

Johnston (T. H.), 1918. - Notes on certain entozoa of rats and mice. Proc. Roy. Soc. Queensland, 30, 53-78.

LANE (C.), 1923. - Some strongylata. Parasitology, 15, 348-364.

Lurz (A.), 1894. - Beobachtungen über die als Taenia nana und flavopunctata bekannten Bandwurmer des Menschen. Zbl. Bakt., 16, 61-67.

Travassos (L.), 1914. - Trichostrongylideos brazileiros (3. nota previa). Brazil Medico, 28, 325-327.

Travassos (L.), 1921. - Contribuicoes para o conhecimento da fauna helmintolojica brasileira. 13. Ensaio monografico da familia Trichostrongylidae Leiper, 1909. Mem. Inst. Osw. Cruz, 13, 1-135.

Tubangur (M.-A), 1931. - Worm parasites of the brown rat (Mus norvegicus) in the Philippine Islands, with special reference to those forms that may be transmitted to human beings. Phil. J. Sci., 46, 537-591.

Yokogawa (S.), 1920. - A new nematode from the rat. J. Parasit., 7, 29-33. 
Yokogawa (S.), 1922. - The development of Heligmosomum muris Yokogawa, a nematode from the intestine of the wild rat. Parasitology, 14, 127-166.

Voelckel (J.), Le Gonidec (G.), Jacobi (J.-C.) et Jehl (R.), 1964. - Helminthes hépatodigestifs parasites de Rattus norvegicus à Marseille. Méd. Trop., 24, 531-536.

Wertheim (G.), 1963. - Helminth parasites of the rat Rattus norvegicus from Haïfa and Tel-Aviv. Bull. Res. Counc. Israel, sect. E, Exp. Med., 10, 125-129.

[Laboratoire de Zoologie (Vers), Muséum National d'Histoire Naturelle] 